\title{
Hemorrhagic cholecystitis and hemobilia: two infrequent complications of systemic lupus erythematosus
}

\author{
Rocío García Pérez ${ }^{1}$, D. Ruiz de Angulo ${ }^{1}$, M.J. López Poveda ${ }^{2}$, B. Febrero Sánchez ${ }^{1}$, D. Navas Carrillo ${ }^{1}$ \\ and Pascual Parrilla Paricio ${ }^{1}$
}

${ }^{1}$ Department of General and Digestive Surgery. ${ }^{2}$ Department of Pathology. Hospital Universitario Virgen de la Arrixaca. Murcia, Spain

\begin{abstract}
Introduction: the patients affected by systemic lupus erythematosus (SLE) often suffer gastrointestinal symptoms. The differential diagnosis should contemplate pathology of the gall bladder. We present the case of a patient with hemorrhagic lithiasic cholecystitis and hemobilia.

Case report: 24 year old female diagnosed with SLE under treatment with Sintrom $\AA$, Dacortin $\AA$ and Dolquine ${ }^{\circledR}$ that presented acute lithiasic cholecystitis and hemobilia with a distal calculus.

Cholecystectomy and aperture of the ductus choledochus were performed allowing to confirm the hemobilia and to extract the calculus.

Discussion: the treatment of cholecystitis in the patients with SLE is controversial due to the fact that most reviewed cases have been solved with cholecystectomy, or in other cases with conservative treatment with corticosteroids. We believe that the presence of cholelithiasis in a patient with SLE with pain on the right hypochondrium and ultrasound confirming the suspicion of cholecystitis demands a surgical treatment since the cause may be vascular, lithiasic or combined. Besides, the possible complications will not respond to pharmaceutical treatment.
\end{abstract}

Key words: Systemic lupus erythematosus. Acute abdominal pain. Hemorrhagic cholecystitis. Hemobilia.

García Pérez R, Ruiz de Angulo D, López Poveda MJ, Febrero Sánchez B, Navas Carrillo D, Parrilla Paricio P. Hemorrhagic cholecystitis and hemobilia: two infrequent complications of systemic lupus erythematosus. Rev Esp Enferm Dig 2011; 103 : 431-433.

Received: $17-11-10$

Accepted: 02-12-10.

Correspondence: Rocío García Pérez. Department of General and Digestive Surgery. Hospital Universitario Virgen de la Arrixaca. Ctra. Madrid-Cartagena, s/n. 30120 El Palmar, Murcia. Spain.

e-mail: araleramsay@me.com

\section{INTRODUCTION}

The patients affected by systemic lupus erythematosus (SLE) often suffer gastrointestinal symptoms due to their condition, but mainly because of the medicines used for their treatment or due to viral or bacterial infections (1). Whenever predominant acute and intense abdominal pain is present, the differential diagnosis (due to its higher prevalence on these patients), should contemplate pancreatitis, appendicitis, intestinal ischemia and pathology of the gallbladder (2). Referring to this last category, acalculous cholecystitis secondary to vasculitis has been widely described in medical literature $(3,4)$. We present the case of a patient with SLE that urgently needed surgical intervention and was diagnosed of hemorrhagic lithiasic cholecystitis and hemobilia, aiming to establish a reference whenever facing these exceptional complications evaluating a patient with SLE with abdominal pain on the right hypochondrium.

\section{CLINICAL CASE}

Our patient is a 24 year old female diagnosed with SLE and antiphospholipid syndrome 10 years ago, under treatment with Sintrom $₫$, Dacortin $₫$ and Dolquine $₫$ consulting over continuous abdominal pain with 24 hours of evolution located on right hypochondrium, not radiated associated to nausea and vomits. In the physical examination, the patient showed painful reaction and voluntary defense at abdominal examination, especially on the right hypochondrium, along with discrete signs of peritoneal irritation with doubtful signs of Murphy's.

The blood tests showed normal value for hematocrit, differential leukocyte count and amylasemia, as well as an initially normal liver test. We requested an abdominal ultrasound where multiple gallbladder lithiasis were observed without any inflammatory signs on the gallbladder wall, as well as a normal bile duct caliber. 
The patient remained under observation suffering increasing pain despite the IV analgesics and suddenly presented jaundice in skin and mucous when explored. The follow up analytics showed a total serum bilirubin level of $6.02 \mathrm{mg} / \mathrm{dl}$, at the expense of a direct bilirubin of 5.83 $\mathrm{mg} / \mathrm{dl}$. Immediate surgery was decided after the patient had a fever peak of $38^{\circ} \mathrm{C}$ besides the previous symptoms.

Through laparoscopic intervention we observed a distended gallbladder with hematomas in its wall. We performed a cholecystectomy and sectioned the cystic duct to introduce a cholangiography catheter. We observed dark blood and clots proceeding from the principal biliary duct and decided to convert to right subcostal laparotomy.

First we realized an intraoperative cholangiography observing a dilated biliary duct and a stop at a distal choledochus level suggesting choledocholithiasis (Fig. 1).

We were able to confirm the hemobilia through the opening of the ductus choledochus and let us discover the presence of a distal calculus that was extracted with a Fogarty catheter. Further on, through the visualization with a choledoscopy, we ruled out the presence of other lithiasis as well as active bleeding in the bile duct, closing it with a Kehr's T tube.

The gallbladder was filled with multiple clots as well as a calculus of $1.5 \mathrm{~cm}$.

The mucous was eroded and friable to contact. The pathologic examination reported hemorrhagic cholecystitis with persistence of medium caliber vessels associated to inflammatory signs (Fig. 2). The postoperatory went by without any further complications and allowed to discharge the patient a week later with a clamped $T$ tube that was removed a month later without any problems.

\section{DISCUSSION}

Abdominal pain is a frequent symptom in patients with SLE. The wide range of pathologies that may cause it com-



Fig. 1. Intraoperatory cholangiography where we can observe a dilated biliary duct and a stop at the distal choledocus level suggesting choledocholithiasis. plicates to give a differential diagnosis due to the fact that the available complementary explorations in the ER room are not conclusive in most occasions. In our case, neither the physical exploration, nor the initial analytics or the ultrasound helped to discover the origin of the pain referred by the patient.

On the other side, even though many gastrointestinal manifestations in the patients with SLE are treated with corticosteroids, others such as ischemia and intestinal perforation have surgical urgency and to delay their practice endangers the patient's life. Therefore, the surgeon must know all the possible complications described in the scientific literature for SLE aiming to direct and apply all the proper diagnose-therapeutical measures in each case.

The hepatic and biliar affectation in the SLE is more common than what might be initially thought due to the fact than even though clinical related manifestations are infrequent, diverse retrospective studies show a range of elevated analytical and histological alterations (5). Therefore, it has been described the presence of hepatic steatosis, active chronic hepatitis, primary biliary cirrhosis, hepatic granuloma and centrilobular necrosis, Budd-Chiari syndrome, etc. On the other hand, the vascular alteration at the vesicular wall level may cause an alithiasic cholecystitis that in extreme cases it may become hemorrhagic (6). In our patient, even though the histological study of the removed gallbladder did not show any strict criteria of vasculitis, we proposed that the persistence of medium caliber vessels associated to inflammatory signs, to biliary lithiasis and to anticlotting treatment, originated a bleeding in the interior of the gallbladder and secondary hemobilia. Possibly the lithiasic obstruction of the biliary ducts increased the pres-

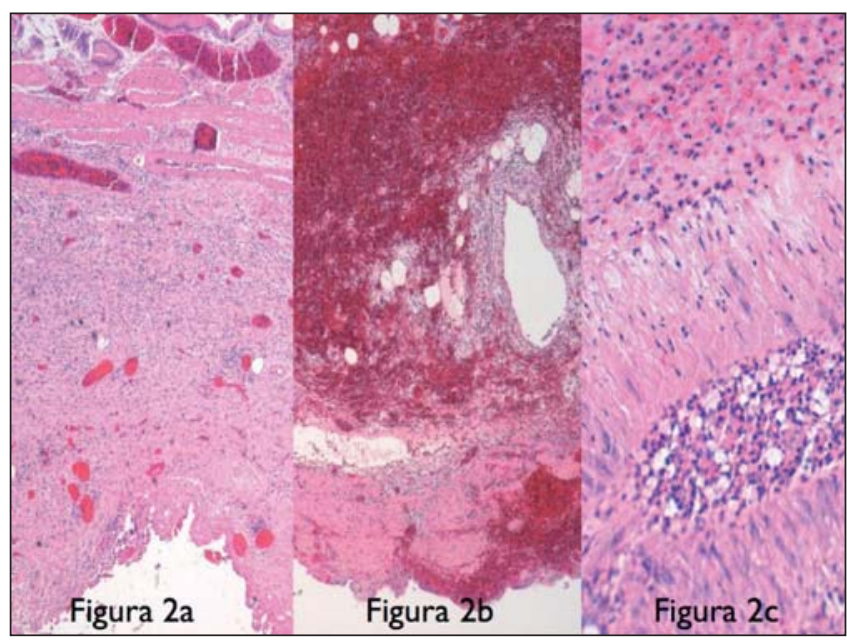

Fig. 2. Gallbladder that shows similar alterations of diffuse character consisting of inflammatory transmural infiltrates by lymphoplasmacytic cells with sources of polinuclear neutrofils leukocytes and vast presence of eosinophils associated to intense vascular congestion (A) with multiple focal areas of recent hemorrhage $(C)$. These areas affect mucous, muscularis externa and serosa without association to parietal necrosis. In C we can appreciate the only vessel with discrete presence of polymorphonuclears in the wall. 
sure on its interior resolving the hemorrhage by clotting and closing the vessel but originated cholestasys and cholangitis.

Even though Rhoton et al. (7) described the case of a patient with lupus that presented hemobilia and obstruction of the main bile duct due to clots, the case we report is the first with these characteristics in which cholelithiasis and choledocholithiasis intervene.

The treatment of cholecystitis in the patients with SLE is controversial due to the fact that most reviewed cases have been solved with cholecystectomy, or in other cases with conservative treatment with corticosteroids (8). We think that the presence of cholelithiasis in a patient with SLE, pain on the right hypochondrium and analytic-ultrasound suspicion of cholecystitis demands a surgical treatment since the cause may be vascular, lithiasic or combined (9). Besides, the possible complications, as the ones described in this case, will be solved during the proper intervention and will not respond to pharmaceutical treatment for sure. To resume, even though we performed a laparotomy to explore the main biliary duct, we believe that to perform a laparoscopy on these patients is useful as a diagnostic tool as well as a therapeutic one according to the experience of the surgeon (10). Minimizing the surgical aggression is even more important in patients with chronic affections and intrinsic or pharmaceutical alterations of the clotting process such as in patients with SLE.

\section{REFERENCES}

1. Tian XP, Zhang X. Gastrointestinal involvement in systemic lupus erythematosus: insight into pathogenesis, diagnosis and treatment. World J Gastroenterol 2010;16:2971-7.

2. Vergara-Fernandez O, Zeron-Medina J, Mendez-Probst C, SalgadoNesme N, Borja-Cacho D, Sanchez-Guerrero J, et al. Acute abdominal pain in patients with systemic lupus erythematosus. J Gastrointest Surg 2009; $13: 1351-7$.

3. Swanepoel CR, Floyd A, Allison H, Learmonth GM, Cassidy MJ, Pascoe MD. Acute acalculous cholecystitis complicating systemic lupus erythematosus: case report and review. Br Med J (Clin Res Ed) 1983;286:251-2.

4. Mendonça JA, Marques-Neto JF, Prando P, Appenzeller S. Acute acalculous colecistitis in juvenile systemic lupus erythematosus. Lupus 2009;18:561-3.

5. Runyon BA, LaBrecque DR, Anuras S. The spectrum of liver disease in systemic lupus erythematosus. Report of 33 histologically-proved cases and review of the literature. Am J Med 1980;69:187-94.

6. Raijman I, Schrager M. Hemorrhagic acalculous cholecystitis in systemic lupus erythematosus. Am J Gastroenterol 1989;84:445-7.

7. Rhoton AJ, Gilliam JH, Geisinger KR. Hemobilia in systemic lupus erythematosus. South Med J 1993;86:1049-51.

8. Kamimura T, Mimori A, Takeda A, Masuyama J, Yoshio T, Okazaki $\mathrm{H}$, et al. Acute acalculous colecistitis in systemic lupus erythematosus: a case report and review of the literature. Lupus 1998;7:361-3.

9. De-Leon-Bojorge B, Zaltzman-Girsevich S, Ortega-Salgado A, Prieto-Patron A, Córdoba-Córdoba R, Yamazaki-Nakashimada M. Thrombotic microangiopathy involving the gallbladder as an unusual manifestation of systemic lupus erythematosus and antiphospholipid syndrome: Case report and review of the literature. World J Gastroenterol 2006; 12:7206-9.

10. Gobbi S, Sarli L, Violi V, Roncoroni L. Laparoscopically assisted treatment of acute abdomen in systemic lupus erythematosus. Surg Endosc 2000;14:1085-6. 